\title{
Suture locking of isolated internal locking knotless suture anchors is not affected by bone quality
}

This article was published in the following Dove Press journal:

Open Access Journal of Sports Medicine

22 June 2015

Number of times this article has been viewed

\author{
Jarret M Woodmass' \\ Graeme Matthewson' \\ Yohei Ono',2 \\ Aaron J Bois' \\ Richard S Boorman' \\ Ian KY Lo' \\ Gail MThornton ${ }^{1,3}$
}

'Department of Surgery, Section of Orthopaedic Surgery, McCaig Institute for Bone and Joint Health, University of Calgary, Calgary, $A B$,

Canada; ${ }^{2}$ Department of Orthopaedic Surgery, Nagoya University Graduate School of Medicine, Nagoya, Japan;

${ }^{3}$ Department of Orthopaedics, University of British Columbia,

Vancouver, BC, Canada
Correspondence: Gail M Thornton McCaig Institute for Bone and Joint Health, Department of Surgery, University of Calgary, 3AI 8 Health Research Innovation Centre, 3280 Hospital Drive NW, Calgary, $\mathrm{AB}, \mathrm{T} 2 \mathrm{~N} 4 \mathrm{Z6}$, Canada

$\mathrm{Tel}+\mathrm{I} 4032202296$

$\mathrm{Fax}+\mathrm{I} 4032108197$

Email gail.thornton@ucalgary.ca
Purpose: The purpose of this study was to evaluate the mechanical performance of different suture locking mechanisms including: i) interference fit between the anchor and the bone (eg, $4.5 \mathrm{~mm}$ PushLock, $5.5 \mathrm{~mm}$ SwiveLock), ii) internal locking mechanism within the anchor itself (eg, $5.5 \mathrm{~mm}$ SpeedScrew), or iii) a combination of interference fit and internal locking (eg, 4.5 mm MultiFIX P, $5.5 \mathrm{~mm}$ MultiFIX S).

Methods: Anchors were tested in foam blocks representing normal (20/8 foam) or osteopenic ( $8 / 8$ foam) bone, using standard suture loops pulled in-line with the anchor to isolate suture locking. Mechanical testing included cyclic testing for 500 cycles from $10 \mathrm{~N}$ to $60 \mathrm{~N}$ at $60 \mathrm{~mm} / \mathrm{min}$, followed by failure testing at $60 \mathrm{~mm} / \mathrm{min}$. Displacement after 500 cycles at $60 \mathrm{~N}$, number of cycles at $3 \mathrm{~mm}$ displacement, load at $3 \mathrm{~mm}$ displacement, and maximum load were evaluated.

Results: Comparing $8 / 8$ foam to $20 / 8$ foam, load at $3 \mathrm{~mm}$ displacement and maximum load were significantly decreased $(P<0.05)$ with decreased bone quality for anchors that, even in part, relied on an interference fit suture locking mechanism (ie, $4.5 \mathrm{~mm}$ PushLock, $5.5 \mathrm{~mm}$ SwiveLock, 4.5 mm MultiFIX P, 5.5 mm MultiFIX S). Bone quality did not affect the mechanical performance of $5.5 \mathrm{~mm}$ SpeedScrew anchors which have an isolated internal locking mechanism.

Conclusion: The mechanical performance of anchors that relied, even in part, on interference fit were affected by bone quality. Isolated internal locking knotless suture anchors functioned independently of bone quality. Anchors with a combined type (interference fit and internal locking) suture locking mechanism demonstrated similar mechanical performance to isolated internal locking anchors in osteopenic foam comparing similar sized anchors.

Clinical relevance: In osteopenic bone, knotless suture anchors that have an internal locking mechanism (isolated or combined type) may be advantageous for secure tendon fixation to bone.

Keywords: suture locking, knotless suture anchors, bone quality

\section{Introduction}

Rotator cuff tears have a high prevalence among the aging population affecting over half of individuals over the age of 60 years. ${ }^{1}$ While surgical treatment of rotator cuff tears is generally less favorable in patients of increasing age, surgery remains an option for patients who suffer from either an acute injury or a chronic, symptomatic full-thickness tear. ${ }^{2}$ Traditionally, rotator cuff tears have been repaired using an open or mini-open technique. However, over the past decade, there has been a gradual shift in repairing rotator cuff tears arthroscopically rather than by an open procedure or mini-open approach. ${ }^{3}$ Arthroscopic advances now permit even massive tears to be routinely repaired arthroscopically. While many different repair techniques can be used, 
arthroscopic rotator cuff repair commonly utilizes suture anchor-based constructs for tendon fixation to bone. ${ }^{4}$

Conventional suture anchor-based constructs have demonstrated multiple modes of failure as previously defined by Barber et $\mathrm{al}^{5}$ including anchor pullout (when the anchor pulls out of the bone), suture breakage (when the suture breaks in two) and eyelet breakage (when an intact suture pulls out of an anchor eyelet). Additionally, knot slippage and suture failure through the tendon have been shown to play a role in the failure of suture anchor-based constructs. ${ }^{6}$ More recently, knotless suture anchors have been introduced to reduce the technical demands and challenges associated with arthroscopic knot tying. ${ }^{7}$ Other proposed advantages of knotless suture anchors include avoiding subacromial impingement via knot omission, ${ }^{8}$ reducing the necessity of surgical exposure, reducing the length of surgery as well as potentially having superior mechanical performance. ${ }^{9,10}$ When compared with conventional anchors, knotless suture anchors exhibited similar or greater loads in failure tests but similar or greater displacements in cyclic tests. ${ }^{5,8,11,12}$ Conventional and knotless suture anchors have also demonstrated equivalent clinical outcomes during Bankart repairs. ${ }^{13}$

The introduction of knotless suture anchors has demonstrated other potential modes of failure than those identified for conventional suture anchors. By definition, knotless suture anchors obtain loop and knot security ${ }^{14}$ in some alternative manner to tying a knot; thus, suture slippage, or the loss of suture knot security within the anchor construct itself, may also occur. Wieser et $\mathrm{al}^{15}$ demonstrated that, under load, suture slippage of knotless suture anchors occurred at lower loads than anchor pullout and was the weakest link in the anchor-suture system.

Various methods of obtaining suture locking for knot security have been utilized in different anchor designs. In general, the mechanisms of suture locking may be categorized as suture fixation between the anchor and the bone (interference fit), suture fixation within the anchor itself (internal locking), or suture fixation from a combination of interference fit and internal locking. Clearly, the mechanism of suture locking may be a factor that contributes to construct failure via suture slippage. In particular, knotless suture anchor designs which incorporate a suture locking mechanism that relies on robust bone quality (eg, interference fit) may be adversely affected when implanted in osteopenic bone. To our knowledge only a single study has assessed the mechanical performance of knotless suture anchors in both healthy and osteopenic bone. While the purpose of the Pietschmann et $\mathrm{al}^{10}$ study was to examine the effect of bone mineral density (BMD) on anchor-to-bone fixation mechanisms categorized as force-fit (anchor retained by friction force) and form-fit (anchor retained by change in form after deployment), their findings led to the speculation that knotless suture anchors with suture-to-anchor fixation that depended upon interference between the bone and the anchor were affected by BMD.

The purpose of this study was to evaluate the mechanical performance and failure modes of different knotless suture anchors, with different suture locking mechanisms, tested in synthetic foam representing normal and osteopenic bone. The suture locking mechanisms tested were: i) interference fit between the anchor and the bone with the suture external to the anchor (eg, $4.5 \mathrm{~mm}$ PushLock, $5.5 \mathrm{~mm}$ SwiveLock; Arthrex Inc., Naples, FL, USA), ii) internal locking mechanism with the suture internal to the anchor (eg, $5.5 \mathrm{~mm}$ SpeedScrew; ArthroCare Corp., Austin, TX, USA), or iii) combination of interference fit and internal locking (eg, 4.5 mm MultiFIX P, 5.5 mm MultiFIX S; ArthroCare Corp.). We hypothesized that the mechanical performance of knotless suture anchors which rely on an isolated internal locking mechanism will not be affected by bone quality but those which rely on a combined interference fit and internal locking mechanism will be affected by bone quality.

\section{Methods}

Different anchors were utilized to represent the different suture locking mechanisms: i) interference fit between the anchor and the bone (eg, $4.5 \mathrm{~mm}$ PushLock and $5.5 \mathrm{~mm}$ SwiveLock), ii) internal locking mechanism within the anchor itself (eg, $5.5 \mathrm{~mm}$ SpeedScrew), iii) combination of interference fit and internal locking (eg, $4.5 \mathrm{~mm}$ MultiFIX P and $5.5 \mathrm{~mm}$ MultiFIX S). Individual anchors were tested in individual polyurethane foam bone blocks (General Plastics Manufacturing Co., Tacoma, WA, USA) representing normal and osteopenic bone. Normal bone was represented by $20 / 8$ foam which had a $3 \mathrm{~mm} 20$ pcf layer laminated on an $8 \mathrm{pcf}$ block, and osteopenic bone was represented by $8 / 8$ foam which was an 8 pcf block. Five samples of each anchor and foam combination were tested. All anchors were tested using a standard suture loop (40.5 mm length) pulled in-line with the anchor to isolate suture locking. Anchors were tested with the suture supplied by the manufacturer: \#2 FiberWire suture for $4.5 \mathrm{~mm}$ PushLock and $5.5 \mathrm{~mm}$ SwiveLock anchors (Arthrex Inc.) and \#2 MagnumWire suture for $5.5 \mathrm{~mm}$ SpeedScrew, $4.5 \mathrm{~mm}$ MultiFIX P and $5.5 \mathrm{~mm}$ MultiFIX S anchors (ArthroCare Corp.).

Mechanical testing was performed using a Lloyd LRX Plus with a 1,000 N load cell and data were collected using 
NEXYGEN Plus 3.0 software (Lloyd Materials Testing, West Sussex, UK). Mechanical testing included preloading, cyclic testing, and failure testing. Preloading to $10 \mathrm{~N}$ was performed at $60 \mathrm{~mm} / \mathrm{min}$ and the preload of $10 \mathrm{~N}$ was held for 10 seconds. Cyclic testing was 500 cycles at $60 \mathrm{~mm} / \mathrm{min}$ between $10 \mathrm{~N}$ and $60 \mathrm{~N} .{ }^{16}$ Failure testing was performed at $60 \mathrm{~mm} / \mathrm{min} .{ }^{17}$

Displacement after 500 cycles at $60 \mathrm{~N}$ was defined as the increase in displacement from the peak $(60 \mathrm{~N})$ of the first cycle to the peak of the 500th cycle. A $3 \mathrm{~mm}$ displacement was defined as $3 \mathrm{~mm}$ displacement relative to the peak of the first cycle. The number of cycles at $3 \mathrm{~mm}$ displacement and the load at $3 \mathrm{~mm}$ displacement were recorded. If a $3 \mathrm{~mm}$ displacement occurred after the maximum load was detected during failure testing, then the maximum load was used as the load at $3 \mathrm{~mm}$ displacement. If failure occurred during failure testing, then maximum load was the maximum load reached during failure testing. If failure occurred during cyclic testing, then maximum load was the maximum load reached during cyclic testing.
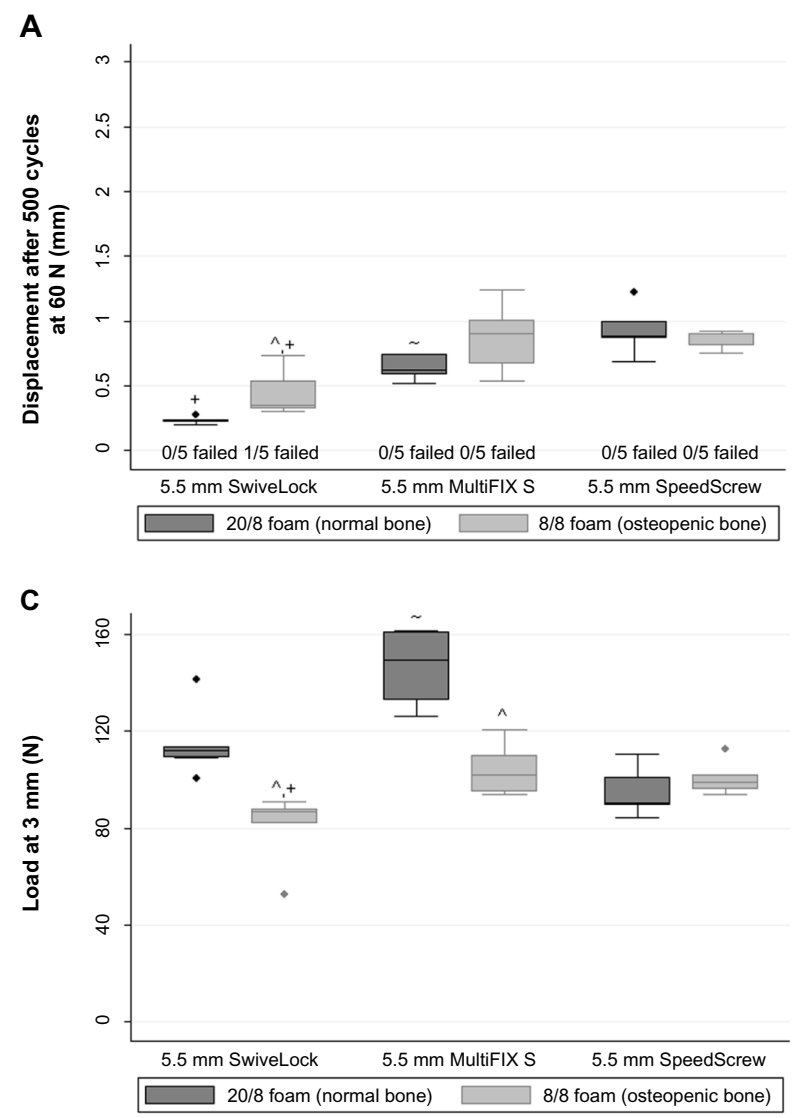

A sample of four was sufficient to detect a difference in the means of $30 \mathrm{~N}$ with a standard deviation of $15 \mathrm{~N}$ (alpha=0.05 and beta $=0.20$ ). Data were analyzed using analysis of variance (ANOVA) with linear contrasts when normally distributed or Kruskal-Wallis test with Conover post hoc analysis when not normally distributed. The numbers of failures during cyclic loading were compared using Fisher's exact test.

\section{Results}

\section{Anchors - $5.5 \mathrm{~mm}$}

In 20/8 foam (normal bone), all of the $5.5 \mathrm{~mm}$ anchors completed cyclic loading without failing (Figure $1 \mathrm{~A}$ to $\mathrm{D}$ and Table 1). Displacement after 500 cycles at $60 \mathrm{~N}$ was significantly different comparing all three anchors to each other: $5.5 \mathrm{~mm}$ SwiveLock, $5.5 \mathrm{~mm}$ MultiFIX S, and $5.5 \mathrm{~mm}$ SpeedScrew $(P<0.05$; Figure 1A). Load at $3 \mathrm{~mm}$ displacement (Figure 1C) and maximum load (Figure 1D) were greater for MultiFIX S compared with SwiveLock and SpeedScrew anchors $(P<0.05)$.
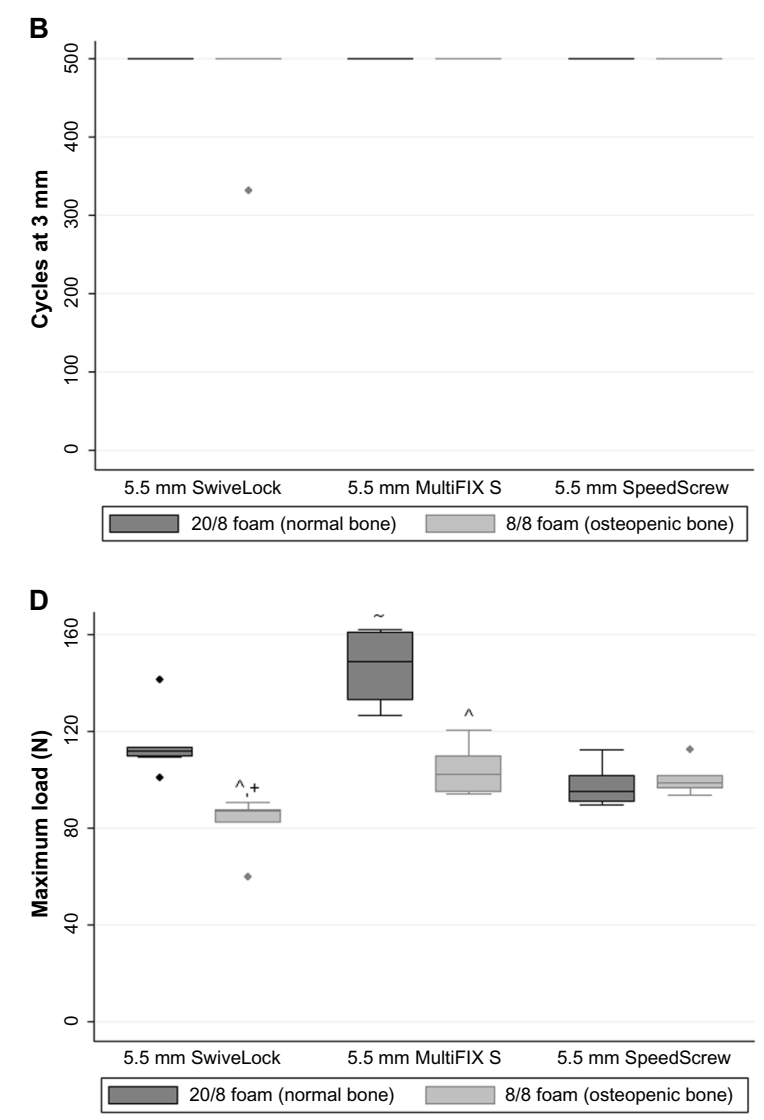

Figure I Mechanical performance of $5.5 \mathrm{~mm}$ SwiveLock (interference fit), $5.5 \mathrm{~mm}$ MultiFIX S (interference fit and internal locking) and $5.5 \mathrm{~mm}$ SpeedScrew (internal locking) anchors.

Notes: (A) Displacement after 500 cycles at $60 \mathrm{~N}$, (B) cycles at $3 \mathrm{~mm}$, (C) load at $3 \mathrm{~mm}$, (D) maximum load. Cycles at $3 \mathrm{~mm}$ were 500 cycles for all but one $5.5 \mathrm{~mm}$ SwiveLock anchor. '8/8 foam (osteopenic bone) different than 20/8 foam (normal bone) for same anchor $(P<0.05)$; ${ }^{+}$SwiveLock different than MultiFIX $S$ and SpeedScrew in same material $(P<0.05)$; - MultiFIX S different than SwiveLock and SpeedScrew in same material $(P<0.05)$. The diamond symbol on the boxplot indicates an outlier. SwiveLock, Arthrex Inc., Naples, FL, USA. MultiFIX S, ArthroCare Corp., Austin, TX, USA. SpeedScrew, ArthroCare Corp. 
Table I Failure mode of $5.5 \mathrm{~mm}$ SwiveLock (interference fit), $5.5 \mathrm{~mm}$ MultiFIX S (interference fit and internal locking) and $5.5 \mathrm{~mm}$ SpeedScrew (internal locking) anchors

\begin{tabular}{|c|c|c|c|c|c|}
\hline Anchor & Material & $\begin{array}{l}\text { Number failed } \\
\text { during cyclic } \\
\text { loading }\end{array}$ & $\begin{array}{l}\text { Failure mode } \\
\text { during cyclic } \\
\text { loading }\end{array}$ & $\begin{array}{l}\text { Number completed } \\
\text { cyclic loading and } \\
\text { failure testing }\end{array}$ & $\begin{array}{l}\text { Failure mode } \\
\text { during failure } \\
\text { testing }\end{array}$ \\
\hline $5.5 \mathrm{~mm}$ SwiveLock & $20 / 8$ foam & $0 / 5$ & $0 / 5$ & $5 / 5$ & $5 / 5$ suture slippage \\
\hline $5.5 \mathrm{~mm}$ MultiFIX S & $20 / 8$ foam & $0 / 5$ & $0 / 5$ & $5 / 5$ & $5 / 5$ suture slippage \\
\hline $5.5 \mathrm{~mm}$ SpeedScrew & 20/8 foam & $0 / 5$ & $0 / 5$ & $5 / 5$ & 5/5 suture slippage \\
\hline $5.5 \mathrm{~mm}$ SwiveLock & $8 / 8$ foam & $\mathrm{I} / 5$ & I/5 suture slippage & $4 / 5$ & 4/5 suture slippage \\
\hline $5.5 \mathrm{~mm}$ MultiFIX S & $8 / 8$ foam & $0 / 5$ & $0 / 5$ & $5 / 5$ & $\begin{array}{l}\text { 4/5 anchor pullout } \\
\text { I/5 suture slippage }\end{array}$ \\
\hline $5.5 \mathrm{~mm}$ SpeedScrew & $8 / 8$ foam & $0 / 5$ & $0 / 5$ & $5 / 5$ & $5 / 5$ suture slippage \\
\hline
\end{tabular}

Notes: SwiveLock, Arthrex Inc., Naples, FL, USA; MultiFIX S, ArthroCare Corp., Austin, TX, USA; SpeedScrew, ArthroCare Corp.

In $8 / 8$ foam (osteopenic bone), one of five SwiveLock anchors failed during cyclic loading; whereas, none of the MultiFIX S or SpeedScrew anchors failed during cyclic loading (Figure 1A and Table 1). Displacement after 500 cycles at $60 \mathrm{~N}$, load at $3 \mathrm{~mm}$ displacement and maximum load were lower for SwiveLock compared to SpeedScrew and MultiFix $\mathrm{S}$ anchors $(P<0.05$; Figure 1A, $\mathrm{C}$ and $\mathrm{D})$.

Comparing osteopenic foam to normal foam, SwiveLock anchors had increased displacement after 500 cycles at $60 \mathrm{~N}$, decreased load at $3 \mathrm{~mm}$ displacement and decreased maximum load with decreased bone quality $(P<0.05$; Figure 1A , C and D). Comparing $8 / 8$ foam to $20 / 8$ foam, MultiFIX S anchors had decreased load at $3 \mathrm{~mm}$ displacement and decreased maximum load with decreased bone quality $(P<0.05$; Figure $1 \mathrm{C}$ and D). SpeedScrew anchors exhibited similar mechanical performance in normal and osteopenic foam (Figure 1).

The failure mode of the $5.5 \mathrm{~mm}$ SpeedScrew anchors was suture slippage during failure testing regardless of bone quality (Table 1). While all of the $5.5 \mathrm{~mm}$ SwiveLock anchors failed via suture slippage, one of the five anchors tested in $8 / 8$ foam failed during cyclic loading with all other anchors failing during failure testing (Figure 1A and Table 1). In 20/8 foam, all five of the $5.5 \mathrm{~mm}$ MultiFIX $\mathrm{S}$ anchors failed via suture slippage during failure testing; whereas, in $8 / 8$ foam, one anchor failed via suture slippage and four anchors failed via anchor pullout during failure testing (Table 1).

\section{Anchors - $4.5 \mathrm{~mm}$}

In 20/8 foam (normal bone), all of the $4.5 \mathrm{~mm}$ anchors completed cyclic loading without failing (Figure 2A to D and Table 2). Maximum load was greater for MultiFIX $P$ than PushLock anchors in 20/8 foam ( $P<0.05$; Figure 2D). In $8 / 8$ foam (osteopenic bone), all of the $4.5 \mathrm{~mm}$ PushLock and $4.5 \mathrm{~mm}$ MultiFIX $P$ anchors failed during cyclic loading (Figure 2A and Table 2).
Comparing osteopenic foam to normal foam, PushLock and MultiFIX P anchors exhibited significantly more failures during cyclic loading, decreased number of cycles to reach $3 \mathrm{~mm}$ displacement, decreased load at $3 \mathrm{~mm}$ displacement, and decreased maximum load with decreased bone quality $(P<0.05$; Figure 2 and Table 2$)$.

In 20/8 foam, all of the $4.5 \mathrm{~mm}$ PushLock anchors failed via anchor pullout during failure testing; whereas, in $8 / 8$ foam, all of the PushLock anchors failed during cyclic loading with three failing via anchor pullout and two failing via suture slippage (Figure 2A and Table 2). In $8 / 8$ foam, all of the $4.5 \mathrm{~mm}$ MultiFIX $\mathrm{P}$ anchors failed via anchor pullout during cyclic loading but in 20/8 foam all of the MultiFIX P anchors failed during failure testing with three failing via suture slippage and two failing via anchor pullout (Table 2).

\section{Discussion}

Consistent with our hypothesis, the anchor that utilized a completely internal locking suture locking mechanism exhibited consistent mechanical performance in both normal and osteopenic foam. Although anchors that used a combined type of suture fixation (interference fit and internal locking) were affected by bone quality with decreased maximum load in osteopenic foam compared with normal foam, the maximum load of the combined type anchors and isolated internal locking anchors was similar in osteopenic foam. Thus, it appears that even a component of internal suture locking may be protective against changes in bone quality.

While all anchors performed well in normal foam, the four anchors $(5.5 \mathrm{~mm}$ SwiveLock, $5.5 \mathrm{~mm}$ MultiFIX S, $4.5 \mathrm{~mm}$ PushLock, $4.5 \mathrm{~mm}$ MultiFIX P) that utilized interference fit suture locking mechanisms were significantly affected by bone quality having comparatively poorer performance in osteopenic foam. Each of these interference fit anchors exhibited decreased load at $3 \mathrm{~mm}$ displacement and decreased maximum load with decreased bone 
A

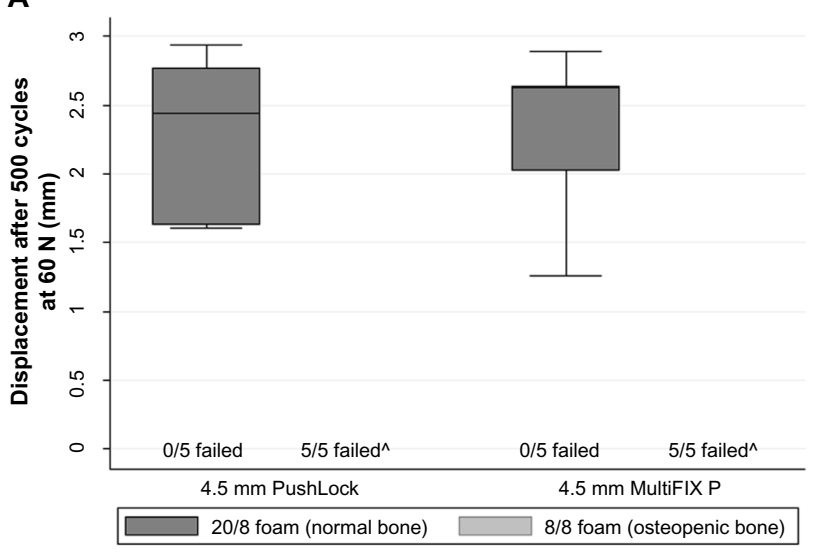

C

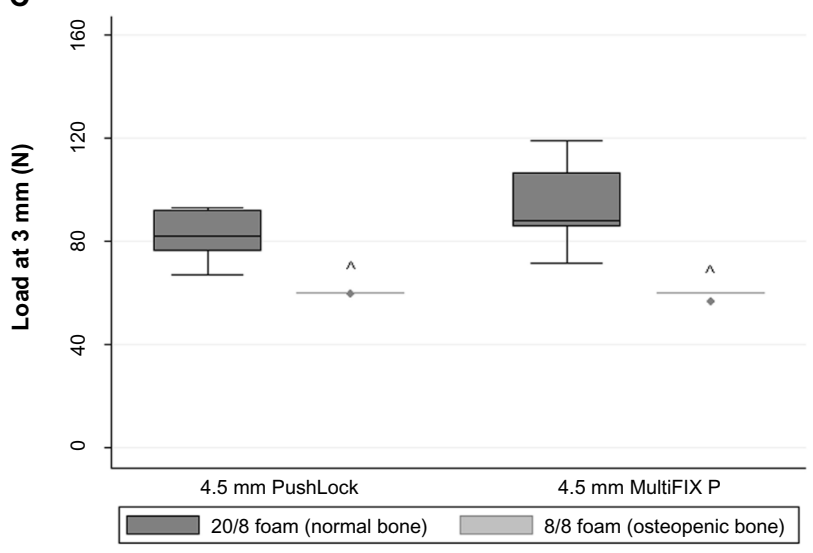

B

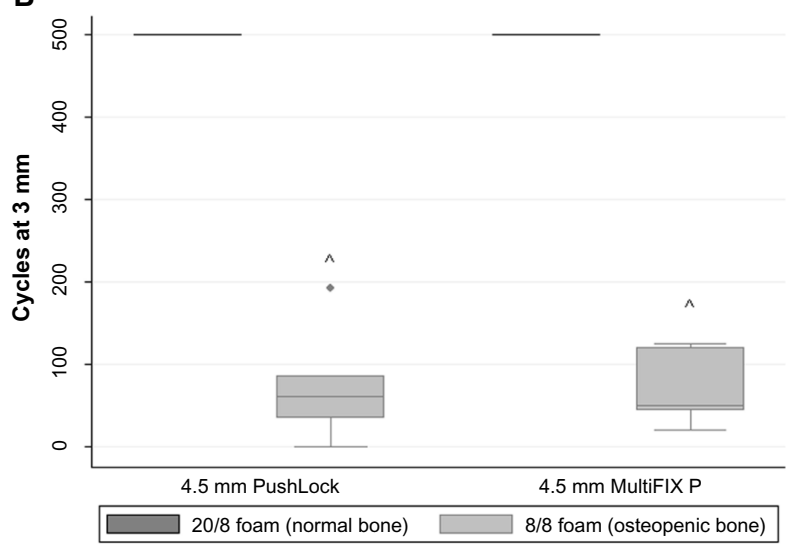

D

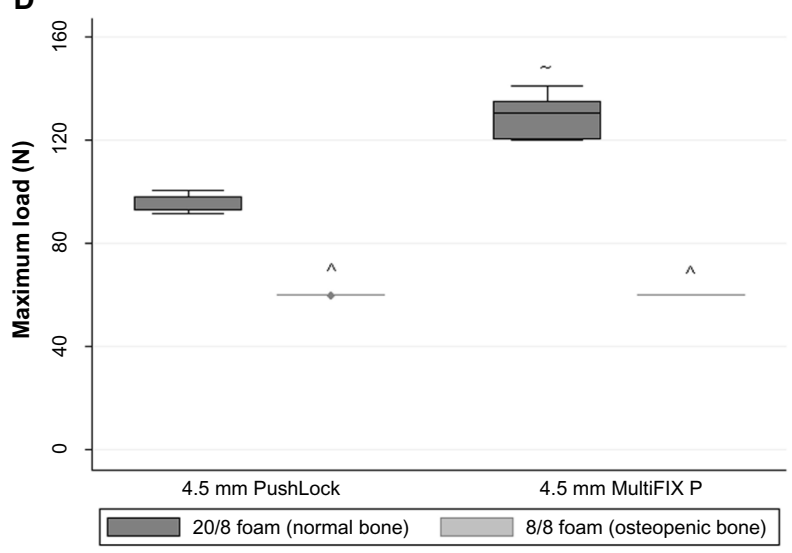

Figure 2 Mechanical performance of $4.5 \mathrm{~mm}$ PushLock (interference fit) and $4.5 \mathrm{~mm}$ MultiFIX P (interference fit and internal locking) anchors.

Notes: (A) Displacement after 500 cycles at $60 \mathrm{~N}$, (B) cycles at $3 \mathrm{~mm},(\mathbf{C})$ load at $3 \mathrm{~mm}$, (D) maximum load. ^ $8 / 8$ foam (osteopenic bone) different than 20/8 foam (normal bone) for same anchor $(P<0.05)$; - MultiFIX $P$ different than PushLock in same material $(P<0.05)$. The diamond symbol on the boxplot indicates an outlier. PushLock, Arthrex Inc., Naples, FL, USA; MultiFIX P, ArthroCare Corp., Austin, TX, USA.

quality. Also, $5.5 \mathrm{~mm}$ SwiveLock (interference fit) anchors exhibited increased displacement after 500 cycles at $60 \mathrm{~N}$ with decreased bone quality. Additionally, $4.5 \mathrm{~mm}$ PushLock (interference fit) anchors and $4.5 \mathrm{~mm}$ MultiFIX P (interference fit and internal locking) anchors had more failures during cyclic loading and decreased number of cycles at $3 \mathrm{~mm}$ displacement with decreased bone quality. In contrast, the one anchor (5.5 mm SpeedScrew) that utilized a completely internal locking suture locking mechanism exhibited consistent mechanical performance in both normal and osteopenic foam. Interestingly, when evaluated in osteopenic foam ( $8 / 8$ foam), the anchor that utilized a combined interference fit and internal locking suture locking mechanism (5.5 mm MultiFIX S) exhibited similar maximum load to the anchor that utilized an isolated internal locking mechanism (5.5 mm SpeedScrew).

Table 2 Failure mode of $4.5 \mathrm{~mm}$ PushLock (interference fit) and $4.5 \mathrm{~mm}$ MultiFIX P (interference fit and internal locking) anchors

\begin{tabular}{|c|c|c|c|c|c|}
\hline Anchor & Material & $\begin{array}{l}\text { Number failed } \\
\text { during cyclic } \\
\text { loading }\end{array}$ & $\begin{array}{l}\text { Failure mode } \\
\text { during cyclic } \\
\text { loading }\end{array}$ & $\begin{array}{l}\text { Number completed } \\
\text { cyclic loading and } \\
\text { failure testing }\end{array}$ & $\begin{array}{l}\text { Failure mode } \\
\text { during failure } \\
\text { testing }\end{array}$ \\
\hline $4.5 \mathrm{~mm}$ PushLock & $20 / 8$ foam & $0 / 5$ & $0 / 5$ & $5 / 5$ & $5 / 5$ anchor pullout \\
\hline $4.5 \mathrm{~mm}$ MultiFIX P & $20 / 8$ foam & $0 / 5$ & $0 / 5$ & $5 / 5$ & $\begin{array}{l}2 / 5 \text { anchor pullout } \\
3 / 5 \text { suture slippage }\end{array}$ \\
\hline $4.5 \mathrm{~mm}$ PushLock & $8 / 8$ foam & $5 / 5^{\wedge}$ & $\begin{array}{l}3 / 5 \text { anchor pullout } \\
2 / 5 \text { suture slippage }\end{array}$ & $0 / 5$ & $0 / 5$ \\
\hline $4.5 \mathrm{~mm}$ MultiFIX P & $8 / 8$ foam & $5 / 5^{\wedge}$ & $5 / 5$ anchor pullout & $0 / 5$ & $0 / 5$ \\
\hline
\end{tabular}

Notes: ^8/8 foam (osteopenic bone) different than 20/8 foam (normal bone) for same anchor ( $<<0.05)$; PushLock, Arthrex Inc., Naples, FL, USA; MultiFIX P, ArthroCare Corp., Austin, TX, USA. 
Suture anchor-based constructs rely on a number of different factors for secure tendon fixation to bone (eg, anchor fixation in bone, knot security, loop security, suture fixation to tendon). Of these, the bone-anchor interface is especially important in elderly patients as anchor pullout and loosening has been shown to increase with decreasing BMD. ${ }^{18,19}$ Anchor pullout leads to gap formation that is especially concerning in elderly patients who have decreased vascular supply, poor soft tissue quality, and potentially a lower healing capacity. ${ }^{9,20}$ This has led some researchers to question the use of suture anchors in patients with osteopenic bone, and instead have recommended open trans-osseous repair techniques. However, Pietschmann et $\mathrm{al}^{21}$ found that pullout strengths of trans-osseous sutures were not greater than suture anchors in either normal or osteopenic bone and suggested that osteopenic bone was not a valid reason to perform an open trans-osseous repair.

With the increasing use of anchors for arthroscopic repairs, advances have been made in anchor design. In 1999, Mitek launched the first knotless suture anchor for use in Bankart repairs. ${ }^{22}$ In recent years, there has been a shift in using knotless suture anchors not just for Bankart repairs and rotator cuff repair of the shoulder but also soft tissue repair throughout the musculoskeletal system. ${ }^{13,23-25}$ Knotless suture anchors have generally demonstrated good biomechanical results. When knotless suture anchors have been compared with conventional suture anchors, they have demonstrated similar or greater loads in failure tests but similar or greater displacements in cyclic tests. ${ }^{5,8,11,12}$ Conventional suture anchors require a knot to be tied to reduce and hold the soft tissue to bone. Although there have been a multitude of studies evaluating the mechanical performance of various knot configurations, ${ }^{26-29}$ arthroscopic knot tying remains one of the more difficult aspects of arthroscopic surgery to master.

The development of knotless suture anchors was in part to obviate the need to tie an arthroscopic knot. Other purported advantages include improved reproducible fixation, low profile repair, and shorter operating time. ${ }^{10,21}$ Knot security is defined as the resistance to suture slippage when a knot is placed under load. In classic knotted repairs, knot security depends on knot configuration, internal interference, slack between throws, and friction of the suture material; ${ }^{14,30}$ however, knotless suture anchors rely on suture anchor design to obtain knot security. Unlike knot tying which is directly under surgeon control, knotless suture anchors rely on suture locking mechanisms for obtaining knot security. Therefore it is critical for surgeons to understand these suture locking mechanisms when performing soft tissue fixation to bone. Unfortunately, these suture locking mechanisms have rarely been evaluated in mechanical studies. ${ }^{15}$

In general, the mechanism of suture locking may be categorized as suture fixation between the anchor and the bone (interference fit), suture fixation within the anchor itself (internal locking), or suture fixation from a combination of interference fit and internal locking. Suture locking designs which rely in part on an interference fit between the anchor and the bone may therefore have poor mechanical performance in osteopenic bone. Poor bone quality has been associated with decreased pullout strength of suture anchors in both cadaveric ${ }^{17-19,31}$ and foam models. ${ }^{17}$

In the current study, the maximum load of the SwiveLock and PushLock anchors was decreased in the $8 / 8$ foam compared to the $20 / 8$ foam which was consistent with the observation of Pietschmann et $\mathrm{a}^{10}$ that SwiveLock and PushLock anchors had decreased maximum failure load when comparing osteopenic humeri with healthy humeri. Importantly, the Pietschmann et al ${ }^{10}$ study was performed to evaluate anchor stability, and anchors were tested at an angle of $135^{\circ}$ to the axis of the humerus. Therefore, under mechanical loading, a number of different factors other than slippage of the suture locking mechanism could be associated with mechanical failure of the anchor constructs, including suture cutting through bone. In the current study, we performed the mechanical testing in-line with the suture anchor in order to isolate suture locking. ${ }^{15}$ This angle of testing minimizes any oblique contact between the suture and the bone, eliminating any potential effect of suture cutting through bone. When evaluated in this fashion, our results suggest that anchors which rely in part on an interference fit between the anchor and the bone may be significantly affected by bone quality and a knotless suture anchor with an internal locking mechanism functions independent of bone quality. This finding supports the previous speculation of Pietschmann et al ${ }^{10}$ that BMD affects knotless suture anchors that depend upon interference between the anchor and bone.

It is important for the surgeon to identify patients with poor bone quality and make appropriate intraoperative adjustment to account for this condition. Patients at risk include those suffering from a chronic rotator cuff tear where osteopenia of the proximal humerus has been shown to occur ${ }^{32}$ and patients of increased age as computed tomography assessment of cadaveric humeri has shown a significant correlation between age and bone quality within the greater tuberosity. ${ }^{33}$ In addition, significant regional differences should be considered as the trabecular microarchitecture of the greater 
tuberosity has greater bone density in the posteromedial region near the articular margin. ${ }^{33}$

Therefore, clinically if the surgeon is faced with osteopenic bone the following alterations to their standard treatment should be considered. First, the anchor size can be increased. In osteopenic foam, all of the $4.5 \mathrm{~mm}$ anchors failed during cyclic loading and only one of the $5.5 \mathrm{~mm}$ anchors, which was an interference fit anchor, failed during cyclic loading. Second, a knotless anchor that utilizes an internal locking mechanism should be considered. This eliminates the reliance on suture fixation at the bone-anchor interface that occurs with interference fit anchors. Third, an attempt should be made to place anchors in the posteromedial aspect of the greater tuberosity, if possible, where regionally improved bone quality is present. ${ }^{33}$ Last, multiple anchors can be used in an attempt to load-share and reduce the individual suture tensions. ${ }^{34}$

\section{Limitations}

While knotless suture anchors are available in several compositions, ${ }^{5}$ we chose to use only polyetheretherketone (PEEK) anchors as these are available in each of the three knotless suture anchor designs being tested (interference fit, internal locking, and combined type). Each anchor was tested with the suture supplied by the manufacturer; thus, the interference fit anchors had a different suture material than the isolated internal locking anchors and the combined type (interference fit and internal locking) anchors. While these components do not represent the breadth of anchor designs available, these components were chosen specifically to compare three types of suture locking mechanisms in two types of foam representing decreasing bone quality. A priori sample size analysis indicated that a sample of four was sufficient to detect a difference in the means of $30 \mathrm{~N}$ with a standard deviation of $15 \mathrm{~N}$ (alpha $=0.05$ and beta $=0.20)$ and no significant difference was found comparing isolated internal locking anchors in normal and osteopenic foam. Post hoc sample size analysis indicated that, for comparison of the maximum load of isolated internal locking anchors in normal and osteopenic foam, the sample size would be 155 which is not likely to be considered practical. Additionally, each anchor was pulled in-line with the anchor to isolate suture locking. This angle of testing minimizes any oblique contact between the suture and the bone, eliminating any potential effect of suture cutting through bone.

Two different foams (20/8 and 8/8) were used to represent normal and osteopenic bone, rather than using cadaveric bone. In order to test anchor designs, many different models have been used including bovine bone, ovine bone, canine bone, fresh frozen human cadaveric bone, and various types of synthetic foam blocks. ${ }^{35}$ Unfortunately not only is disease-free cadaveric bone expensive and difficult to obtain, ${ }^{36}$ but cadaveric bones possess large variations in shape and properties, requiring substantially larger sample sizes in order to obtain statistically significant data. Previous studies have evaluated the mechanical performance of suture anchors tested in foam blocks representing osteopenic bone. ${ }^{17}$ Therefore, in the current study, we used foam blocks to compare the mechanical performance of the various suture locking mechanisms. While the current study demonstrated the principle that a knotless suture anchor which utilized an internal locking mechanism functioned independent of bone density, more studies need to be performed to determine the most common mode of failure, and whether the failure mechanism is dependent upon BMD in cadaveric specimens.

\section{Conclusion}

Knotless suture anchors which rely, even in part, on interference fit between the anchor and the bone were affected by bone quality; whereas, isolated internal locking anchors functioned independently of bone quality. Combined type anchors exhibited decreased maximum load in osteopenic foam compared with normal foam; however, in osteopenic foam, the maximum load was similar comparing isolated internal locking anchors with combined interference fit and internal locking anchors likely due to some protective effect of the internal locking mechanism against changes in bone quality. Thus, if osteopenic bone is suspected, a knotless suture anchor that has an internal locking mechanism (isolated or combined type) may be advantageous for secure tendon fixation to bone.

\section{Disclosure}

IKYL and GMT received an ArthroCare Grant Panel Independent Research Grant. IKYL is a consultant for Arthrex Inc. and ArthroCare Corp. The authors have no other conflicts of interest to disclose.

\section{References}

1. Sher JS, Uribe JW, Posada A, Murphy BJ, Zlatkin MB. Abnormal findings on magnetic resonance images of asymptomatic shoulders. J Bone Joint Surg Am. 1995;77(1):10-15.

2. Pedowitz RA, Yamaguchi K, Ahmad CS, et al. Optimizing the management of rotator cuff problems. J Am Acad Orthop Surg 2011;19(6):368-379

3. Burkhart SS, Lo IK. Arthroscopic rotator cuff repair. J Am Acad Orthop Surg. 2006;14(6):333-346. 
4. Tingart MJ, Apreleva M, Zurakowski D, Warner JJ. Pullout strength of suture anchors used in rotator cuff repair. J Bone Joint Surg Am. 2003; 85(11):2190-2198.

5. Barber FA, Herbert MA, Hapa O, et al. Biomechanical analysis of pullout strengths of rotator cuff and glenoid anchors: 2011 update. Arthroscopy. 2011;27(7):895-905.

6. Cummins CA, Murrell GAC. Mode of failure for rotator cuff repair with suture anchors identified at revision surgery. J Shoulder Elbow Surg. 2003;12(2):128-133.

7. Thal R, Nofziger M, Bridges M, Kim JJ. Arthroscopic bankart repair using knotless or BioKnotless suture anchors: 2- to 7-year results. Arthroscopy. 2007;23(4):367-375.

8. Pietschmann MF, Froehlich V, Ficklscherer A, Wegener B, Jansson V, Müller PE. Biomechanical testing of a new knotless suture anchor compared with established anchors for rotator cuff repair. J Shoulder Elbow Surg. 2008;17(4):642-646.

9. Lewis CW, Schlegel TF, Hawkins RJ, James SP, Turner AS. The effect of immobilization on rotator cuff healing using modified mason-allen stitches: A biomechanical study in sheep. Biomed Sci Instrum. 2001;37: 263-268.

10. Pietschmann MF, Gülecyüz MF, Fieseler S, et al. Biomechanical stability of knotless suture anchors used in rotator cuff repair in healthy and osteopenic bone. Arthroscopy. 2010;26(8):1035-1044.

11. Leedle BP, Miller MD. Pullout strength of knotless suture anchors. Arthroscopy. 2005;21(1):81-85.

12. Brown BS, Cooper AD, McIff TE, Key VH, Toby EB. Initial fixation and cyclic loading stability of knotless suture anchors for rotator cuff repair. J Shoulder Elbow Surg. 2008;17(2):313-318.

13. Ng DZ, Kumar VP. Arthroscopic Bankart repair using knot-tying versus knotless suture anchors: Is there a difference? Arthroscopy. 2014;30(4):422-427.

14. Lo IK, Burkhart SS, Chan KC, Athanasiou K. Arthroscopic knots: Determining the optimal balance of loop security and knot security. Arthroscopy. 2004;20(5):489-502.

15. Wieser K, Farshad M, Vlachopoulos L, Ruffieux K, Gerber C, Meyer DC. Suture slippage in knotless suture anchors as a potential failure mechanism in rotator cuff repair. Arthroscopy. 2012;28(11): 1622-1627.

16. Barber FA, Hapa O, Bynum JA. Comparative testing by cyclic loading of rotator cuff suture anchors containing multiple high-strength sutures. Arthroscopy. 2010;26(9 Suppl):S134-S141.

17. Poukalova M, Yakacki CM, Guldberg RE, et al. Pullout strength of suture anchors: Effect of mechanical properties of trabecular bone. J Biomech. 2010;43(6):1138-1145.

18. Tingart MJ, Apreleva M, Lehtinen J, Zurakowski D, Warner JJ. Anchor design and bone mineral density affect the pull-out strength of suture anchors in rotator cuff repair: Which anchors are best to use in patients with low bone quality? Am J Sports Med. 2004;32(6):1466-1473.

19. Meyer DC, Mayer J, Weber U, Mueller A, Koch PP, Gerber C. Ultrasonically implanted PLA suture anchors are stable in osteopenic bone. Clin Orthop Relat Res. 2006;442:143-148.
20. Hattrup SJ, Ariz S. Rotator cuff repair: Relevance of patient age. J Shoulder Elbow Surg. 1995;4(2):95-100.

21. Pietschmann M, Frohlich V, Ficklscherer A, et al. Pullout strength of suture anchors in comparison with transosseous sutures for rotator cuff repair. Knee Surg Sports Traumatol Arthrosc. 2008;16(5):504-510.

22. Thal R. A knotless suture anchor: Technique for use in arthroscopic Bankart repair. Arthroscopy. 2001;17(2):213-218.

23. Barber FA, Herbert MA. Cyclic loading biomechanical analysis of the pullout strengths of rotator cuff and glenoid anchors: 2013 update. Arthroscopy. 2013;29(5):832-844.

24. Vega J, Golanó P, Pellegrino A, Rabat E, Peña F. All-inside arthroscopic lateral collateral ligament repair for ankle instability with a knotless suture anchor technique. Foot Ankle Int. 2013;34(12):1701-1709.

25. Song HS, Williams GR Jr. All-arthroscopic biceps tenodesis by knotless winding suture. Arthrosc Tech. 2012;1(1):e43-e46.

26. Abbi G, Espinoza L, Odell T, Mahar A, Pedowitz R. Evaluation of 5 knots and 2 suture materials for arthroscopic rotator cuff repair: Very strong sutures can still slip. Arthroscopy. 2006;22(1):38-43.

27. Mahar AT, Moezzi DM, Serra-Hsu F, Pedowitz RA. Comparison and performance characteristics of 3 different knots when tied with 2 suture materials used for shoulder arthroscopy. Arthroscopy. 2006;22(6): 610-614. e1-e2.

28. Hassinger SM, Wongworawat MD, Hechanova JW. Biomechanical characteristics of 10 arthroscopic knots. Arthroscopy. 2006;22(8): 827-832.

29. Baumgarten K, Brodt M, Silva M, Wright R. An in vitro analysis of the mechanical properties of 16 arthroscopic knots. Knee Surg Sports Traumatol Arthrosc. 2008;16(10):957-966.

30. Burkhart SS, Wirth MA, Simonich M, Salem D, Lanctot D, Athanasiou K. Knot security in simple sliding knots and its relationship to rotator cuff repair: How secure must the knot be? Arthroscopy. 2000;16(2): 202-207.

31. Yakacki CM, Poukalova M, Guldberg RE, et al. The effect of the trabecular microstructure on the pullout strength of suture anchors. J Biomech. 2010;43(10):1953-1959.

32. Meyer DC, Fucentese SF, Koller B, Gerber C. Association of osteopenia of the humeral head with full-thickness rotator cuff tears. J Shoulder Elbow Surg. 2004;13(3):333-337.

33. Kirchhoff C, Braunstein V, Milz S, et al. Assessment of bone quality within the tuberosities of the osteoporotic humeral head: relevance for anchor positioning in rotator cuff repair. Am J Sports Med. 2010;38(3):564-569.

34. Kulwicki KJ, Kwon YW, Kummer FJ. Suture anchor loading after rotator cuff repair: effects of an additional lateral row. J Shoulder Elbow Surg. 2010;19(1):81-85.

35. Thompson JD, Benjamin JB, Szivek JA. Pullout strengths of cannulated and noncannulated cancellous bone screws. Clin Orthop Relat Res. 1997;(341):241-249.

36. Szivek J. Synthetic materials and structures used as models for bone. In: An YH, Draughn RA, editors. Mechanical Testing of Bone and The Bone-Implant Interface. Boca Raton: CRC Press, 1999:159-174.
Open Access Journal of Sports Medicine

\section{Publish your work in this journal}

Open Access Journal of Sports Medicine is an international, peer-reviewed, open access journal publishing original research, reports, reviews and commentaries on all areas of sports medicine. The manuscript management system is completely online and includes a very quick and fair peer-review system.

\section{Dovepress}

Visit http://www.dovepress.com/testimonials.php to read real quotes from published authors. 